\title{
Isomer Differentiation in 7, 12-Dimethylbenz $a$ ]anthracene-Pyridine Adducts by Fast Atom Bombardment Tandem Mass Spectrometry
}

\section{G. G. Dolnikowski* and M. L. Gross}

Midwest Center for Mass Spectrometry, Department of Chemistry, University of Nebraska-Lincoln, Lincoln, Nebraska, USA

\section{E. L. Cavalieri}

The Eppley Institute for Research in Cancer, University of Nebraska Medical Center, Omaha, Nebraska, USA

Three isomeric 7,12-dimethylbenz [ a] anthracene-pyridine adduct salts, namely, the 5-Npyridinium-7,12-dimethylbenz $|a|$ anthracene perchlorate, the $7-N$-pyridiniummethylene-12methylbenz [ $a$ ]anthracene picrate, and the 7-methyl-12-N-pyridiniummethylenebenz $[a]$ anthracene picrate, were studied by fast atom bombardment tandem mass spectrometry using high energy collisional-activated dissociation (CAD). The CAD mass spectra of the molecular cations and the ( $\mathrm{M}$ - pyridine $)^{+}$ions allow one to distinguish positional isomers on the basis of daughter ion peak height ratios. The differences in the CAD mass spectra of the $(\mathrm{M}-\text { pyridine })^{+}$ions are probably due in part to formation of isomer-specific fused-ring tropylium ions. (I Am Soc Mass Spectrom 1991, 2, 256-258)

$\mathrm{R}$ adical cations are intermediates in the metabolism $[1,2]$ and covalent binding $[3,4]$ of polycyclic aromatic hydrocarbons (PAH) catalyzed by cytochrome P-450 and peroxidases. Several lines of evidence also suggest that these intermediates, as well as the bay region diol epoxides, play a role in the mechanism of tumor initiation by PAH [4-6]. The structures of the adducts that 7,12-dimethylbenz[a]anthracene (DMBA) forms with DNA are indicative of mechanisms of activation and are critical for judging the relative importance of radical cations and diol epoxides in the mechanism of PAH carcinogenesis.

One of the most potent cancer-causing PAH is DMBA. Recause the structural analysis of DMBAnucleoside adducts is expected to be challenging, research on DMBA-pyridinium adducts as model compounds was undertaken, as is reported here. Three previously synthesized [7] isomeric DMBA-pyridinium adducts of known structure were studied to determine if it is possible to differentiate among them by

\footnotetext{
*Current address: USDA Human Nutritition Research Center on Aging at Tufts University, Boston, Massachusetts, USA.

Address reprint requests to Michael L. Gross, Department of Chemistry, University of Nebraska, Lincoln, NE 68588-0304.
}

using tandem mass spectrometry (MS/MS). This requires locating substituents on aromatic ring systems, which is a classic problem in mass spectrometry [8]. Because all of the $\mathrm{C}-\mathrm{C}$ bonds and all of the $\mathrm{C}-\mathrm{H}$ bonds in aromatic ions have very similar bond energies, and because aromatic ions isomerize rapidly after formation, fragmentation patterns for isomericsubstituted aromatic ions are usually very similar to one another.

The structures of the DMBA-pyridinium adducts were determined previously by nuclear magnetic resonance [7], but if PAH adducts with bases derived from DNA are to be studied either in vitro or in vivo, more sensitive methods for structure determination are necessary. In this study, a fast atom bombardment ionization tandem mass spectrometry (FAB/MS/MS) [9] method has been developed for identifying DMBA-pyridinium adducts.

\section{Experimental Section}

The instrument used for this work was a Kratos MS-50 triple analyzer tandem mass spectrometer, as previously described [10]. It consists of a high resolution MS-I of Nier-Johnson geometry, followed by an electrostatic analyzer as MS-II. Approximately $10 \mathrm{ug}$ of a 
solid sample of each adduct was dissolved directly in approximately $2 \mathrm{uL}$ of dithiothreitol/dithioerythritol matrix. A 6-keV beam of argon atoms desorbed the preformed ions from the viscous liquid matrix. The sample and the matrix were supported in the vacuum chamber $\left(7 \times 10^{-6}\right.$ torr) by a copper probe held at 8 kV. Collision-activated dissociation (CAD) spectra were obtained in MS/MS mode by activating the ions in the third field-free region (between MS-I and MS-II) by collision with helium gas (sufficient to suppress the parent ion beam by $50 \%$ ). By scanning MS-II, CAD spectra were obtained.

\section{Results and Discussion}

Three isomers of the DMBA-pyridinium adducts were investigated: one with pyridine substituted at the 5-position of the ring system and two others, each with pyridine on a different methyl group. The reason for selecting the latter two isomers is that the 7 - and 12-methyl positions of the DMBA radical cation are the most reactive with nucleophiles [6]. Furthermore, the 5-ring pusition is also reactive with potent nucleophiles such as pyridine [7]. Because all of the adducts are salts, a peak corresponding to the preformed molecular cation is found at $m / z 334$ in all three FAB/MS spectra, as is the ( $M-$ pyridine $)^{+}$ion at $m / z$ 255. In mass spectra of the methyl-bound adducts, the fragment ion at $m / z 255$ gives rise to the base peak, and the molecular cation has a relative abundance of $40 \%$. In the mass spectrum of the ringbound adduct, the molecular cation gives rise to the base peak, and the fragment ion at $m / z 255$ has a relative abundance of $\mathbf{1 0 \%}$. Because these adducts are low in molecular weight, the interference from matrix ions is substantial. Therefore, it is difficult to determine from these spectra whether other fragment ion peaks are present. It is clear from the FAB mass spectra that the ring-bound adduct has a stronger $\mathrm{N}-\mathrm{C}$ bond than the methyl-bound adducts because the relative abundance of the $\mathrm{m} / \mathrm{z} 255$ ion formed from the cleavage of that bond is approximately an order of magnitude greater in the methyl-bound adducts than in the ring-bound adduct.

Because the ions derived from the FAB matrix interfere with mass spectra especially at low levels, it is desirable to evaluate the utility of CAD spectra of the molecular cations. Furthermore, identification of DMBA-nucleoside adducts at the low levels characteristic of biological studies will require MS/MS, as was the case for benzo[a]pyrene [3]. The parent ion $\left(\mathrm{M}^{+}\right.$ of $m / z$ 334) was mass-selected with a resolving power of approximately 2500 to remove isobaric interferences due to matrix ions, and the CAD spectra were taken (see Figure 1). The CAD mass spectra of the methylbound adducts (Figure $1 \mathrm{a}$ and $\mathrm{b}$ ) show only two major product ion peaks: one due to the loss of pyridine to give an ion of $m / z 255$, and one due to the loss of $\mathrm{CH}_{3}{ }^{*}$ and pyridine to give an ion of $m / z 240$. The
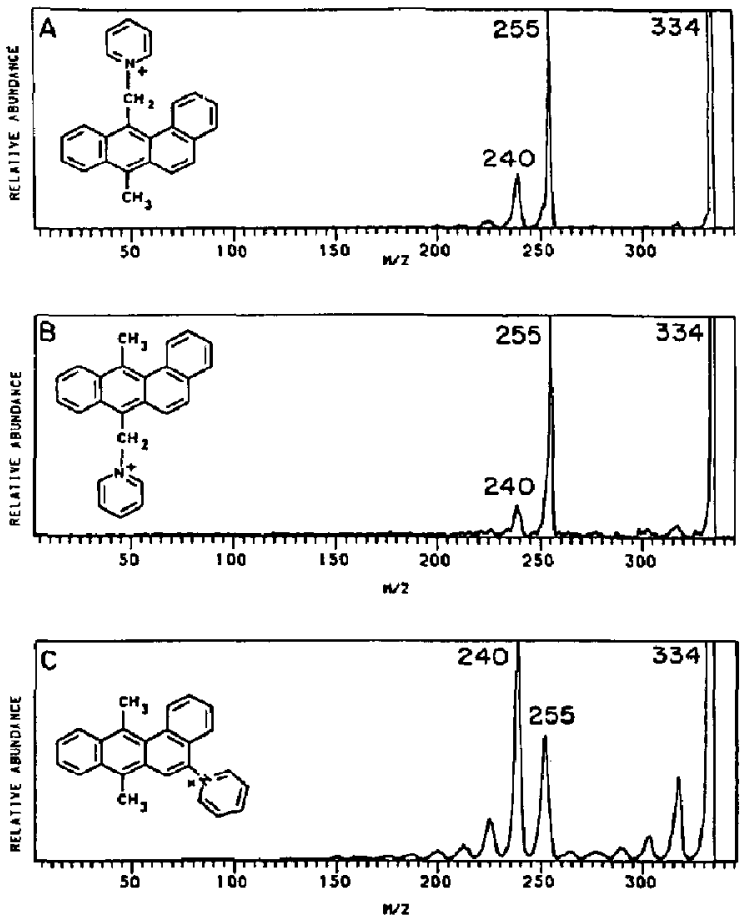

Figure 1. Collision-activated dissociation spectra of the $\mathrm{M}^{+}$ ions $(m / z \quad 334)$ of (a) 7-methyl-12- $N$-pyridiniummethylenebenz[a]anthracene picrate, (b) 7-N-pyridiniummethylene-12methylbenz [ $a$ ]anthracene picrate, and (c) 5-N-pyridinium- $7,12-$ dimethylbenz $[a]$ anthracene perchlorate.

CAD mass spectrum of the ring-bound adduct (Figure 1c) is substantially different showing, in addition to the ions of $m / z 240$ and 255, a whole series of ions formed by serial losses of $13 \mathrm{u}$, which indicates fragmentation of the ring system. Subsequent reinvestigation of the spectra in Figure 1 at higher resolving power with a four-sector instrument indicated that all of the product ion peaks shown in Figure 1 are in fact multiplets. Whereas the ring-bound adduct is easily distinguished from the methyl-bound adducts by the $\mathrm{CAD}$ fragmentation pattern, the two methyl-bound adducts are virtually indistinguishable on the basis of Figure 1 and the FAB mass spectrum.

As mentioned earlier, the major fragment ion in the FAB/MS of the adducts is of $\mathrm{m} / \mathrm{z} 255$, which is formed by the loss of pyridine and is the $(\mathrm{M}-\mathrm{H})^{+}$ ion of DMBA. Collision-activated dissociation mass spectra of methylated aromatic systems rarely differentiate among isomers because tropylium ion formation leads to a symmetrical intermediate in which positional information is lost. Positional information, however, is not lost for the $m / z 255$ ions, as is shown in Figure 2. Fast atom bombardment of the three adducts produces $m / z 255$ fragment ions whose CAD spectra can be used to distinguish the three isomers 

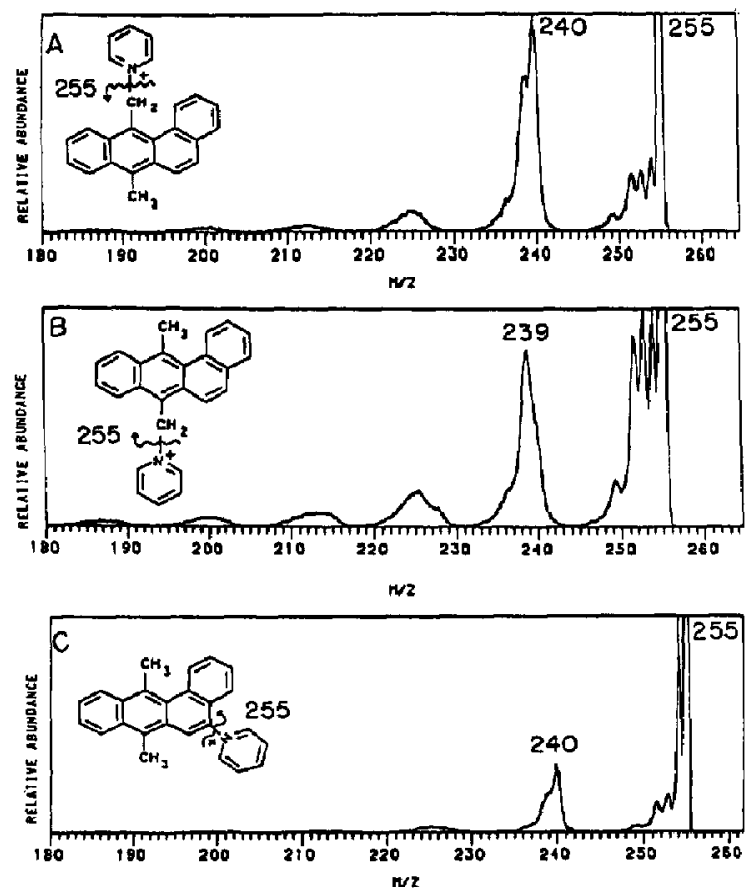

Figure 2. Collision-activated dissociation spectra of the (Mpyridine) ${ }^{+}$ion ( $\left.m / z 255\right)$ of (a) 7 -methyl-12- $N$-pyridiniummethylenebenz [a]anthracene picrate, (b) 7-N-pyridiniummethylene12-methylbenz[a]anthracene picrate, and (c) 5- $\mathrm{N}$-pyridinium7.12-dimethylbenz [ $a$ ] anthracene perchlorate.

on the basis of the ratio of the product ion peak heights at $\mathrm{m} / \mathrm{z} 254$ and 239 . The product ion peak height ratio is $2.3 \pm 0.4$ for the 12-methyl adduct, $0.7 \pm 0.08$ for the 7-methyl adduct, and $0.39 \pm 0.09$ for the 5-ring adduct. The standard deviations quoted here are for seven trials over a period of one year. Increasing the pressure of helium collision gas increases this product ion peak height ratio. It is apparent that control over collision gas pressure is an important factor for the reproducibility of the experiment.

Mixture analysis of these isomers would be difficult by MS/MS because the spectra are qualitatively similar. Thus, we project the need for chromatographic separation prior to or in combination with MS/MS when bioadducts are to be investigated.

Some mechanism must allow the hydrocarbon ion to "remember" where the departed pyridine was attached. Fused-ring tropylium ion formation [11] is known to occur in the $(M-1)^{+}$ions of PAH compounds. We suggest on the basis of the data in Figure 2 that fused-ring tropylium ions with different structures and different CAD spectra form when pyridine is lost from the 7- or 12-methyl position of DMBA (see Scheme I). Although the fused-ring tropylium ions depicted in Scheme I both fragment by loss of $\mathrm{H} \cdot$ and $\mathrm{CH}_{4}$, they preferentially lose either $\mathrm{H} \cdot$ or $\mathrm{CH}_{4}$.

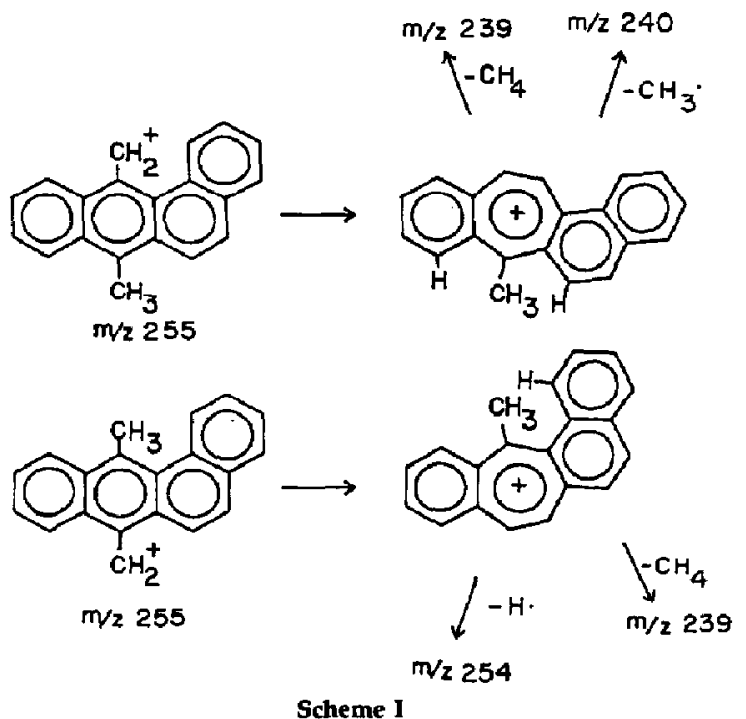

In summary, FAB of DMBA-pyridinium adducts produces fragment ions at $m / z 255$ with fused-ring tropylium ion structures. The CAD spectra of these fragment ions indicate the position at which pyridinium is attached to the DMBA. We expect that FAB of DMBA-nucleoside adducts should produce the same fragment ions, and therefore, the CAD spectra of these fragment ions should reflect the position of DMBA attachment to the base of the nucleoside. Synthesis of DMBA-nucleoside adducts and mass spectral studies are currently under investigation in these laboratories.

\section{References}

1. Cavalieri, E. L.; Rogan, E. G.; Cremonesi, P.; Devanesan, P. D.; Biochem. Pharmacol. 1988, 37, 2173-2182.

2. Cavalieri, E. L.; Devanesan, P. D.; Rogan, E. G. Biochem. Pharmacol. 1988, 37, 2183-2187.

3. Rogan, E. G.; Cavalieri, E. L.; Tibbels, S. R.; Cremonesi, P.; Warner, C. D.; Nagel, D. L,; Tomer, K. B.; Cerny, R. L.; Gross, M. L. J. Am. Chem. Soc. 1988, 110, 4023-4029.

4. Cavalieri, E. L.; Rogan, E. G.; Devanesan, P. D.; Cremonesi, P.; Cerny, R. L.; Gross, M. L.; Bodell, W. J. Biochemistry 1990, 29,4820-4827.

5. Cavalieri, E. L.; Rogan, E. G. Free Radicals in Biology; Pryor, W. A., Ed.; Academic: New York, 1984; Vol. VI, pp. 323-369.

6. Cavalieri, E. L.; Rogan, E. G. Environ. Health Perspect, 1985, 64, 69-84.

7. Cavalieri, E.; Roth, R. I. Org . Chemt 1976, 41, 2679-2684.

8. McLafferty, F. W. Interpretation of Mass Spectra, 3rd ed.; University Science Books: Mill Valley, CA, 1980; pp. $186-189$.

9. Crow, F. W.; Tomer, K. B.; Gross, M. L. Mass Spectrom. Reo. 1983, 2, 47-76.

10. Gross, M. L.; Chess, E. K.; Lyon, P. A.; Crow, F. W.; Evans, S.; Tudge, H. Int. I. Mass Spectrom. Ion Phys. 1982, $42,243-254$.

11. Standley L. J.; Hites, R. A. Org. Mass Spectrom. 1989, 24, 767-772. 